\title{
Review of: "Resistance to a CRISPR-based gene drive at an evolutionarily conserved site is revealed by mimicking genotype fixation"
}

\author{
Gregory Lanzaro ${ }^{1}$ \\ 1 University of California, Davis
}

Potential competing interests: The author(s) declared that no potential competing interests exist.

This gene drive paper presents the results of an experiment that leads the authors to conclude that “...ultra-conservation alone need not be predictive of a site that is refractory to target site resistance".

Gene drive resistance may arise in two ways, when repair at the cleavage site is mediated by NHEJ rather than HDR or as part of standing genetic variation (sgv) in the target population. The authors propose that resistance may be avoided by selecting target sites that are "ultra-conserved". Their use of this concept in the context of the issue addressed here is unfortunate, because it is more appropriate to problems in phylogenetics than in population genetics. Their description of their target site as ultra-conserved when a survey showed variation at $0.6 \%$ is a liberal definition of ultra-conserved. Furthermore, this estimate is itself problematic because it assumes one, that their 2784 mosquitoes sampled across Africa represents a single population and two because they are pooling data across two species, A. gambiae and A. coluzzii. From the standpoint of gene drive resistance, it is more important that the drive target site is not polymorphic within the population being targeted, not across taxa. This addresses both the expectation that drive resistance alleles generated de novo as well as those that may already be present within a population are not selected against.

The major conclusion is that their choice of a target gene was unfortunate because the drive system created a haplo-insufficient genotype with a huge load such that the "fitness costs were severe enough to prevent the gene drive invading at all". They will need to identify additional parameters for target site selection, or they may ultimately get lucky and find a truly germline-restricted female specific haplo-lethal gene or try and make a synthetic one. Alternatively, a way to make gene drive systems work despite the emergence of drive-resistant alleles could be developed. For example, population modification strategies have been shown to better tolerate DRAs, at least in theory.

The authors have done a poor job in referencing important work outside of their own group that is highly relevant to their topic and should be discussed (e.g. Djihinto et al. Res Square 2021, Drury et al. Sci Adv 2017, Lanzaro et al. BioEssays 2021, Marshall et al. Sci Reports 2017, Schmidt et al. Nat Comm. 2020). 
